\title{
Laser processing of protein crystals for native SAD data collection
}

\author{
Ayaka Harada ${ }^{1}$, Naohiro Matsugaki ${ }^{1,2}$, Yoshiaki Kawano ${ }^{3}$, Naoki Matsumoto ${ }^{3,4}$, Masaki \\ Yamamoto $^{3}$, Toshiya Senda ${ }^{1,2}$
}

${ }^{1}$ Structure Biology Research Center, Photon Factory, KEK, ${ }^{2}$ The Grad. Univ. for Advanced Studies, School of High Energy Accelerator Science, ${ }^{3}$ RIKEN/SPring8, ${ }^{4}$ Univ. of Hyogo

Native SAD phasing uses anomalous scattering signals from light atoms such as sulfur and phosphorus in protein crystals. The anomalous signals from these atoms are, however, much weaker than those from heavy atoms that are frequently utilized in protein crystallography. Therefore, high quality data collection is essential for native SAD phasing. Lower energy X-ray is favorable to enhance the anomalous signals but on the other hand X-ray absorption by sample, solvent, and air hinders the diffraction data collection. Here, we present a method minimizing the X-ray absorption; the solvent portion of a mounted frozen crystal is removed or the mounted crystal is spherically shaped by the deep UV laser processing technique ${ }^{1,2}$. In this study, crystals of BphA4 and LigM proteins with various size and shape were used. Diffraction data for native SAD phasing were collected with the crystals in two times before and after the laser processing using the X-ray of $3.7 \mathrm{keV}$ and $4.5 \mathrm{keV}$ at BL-1A of Photon Factory (Tsukuba, Japan), equipped with a helium beam path and a helium cold stream to reduce X-ray absorption by air. The data statistics of the laser-processed crystals were much better: the values of I/sigma(I) and SigAno were significantly increased. The improvement are expected to work advantageously in phase determination by native-SAD method.

1. Kitano, H. et al. Protein Cryocrystallography Using Laser-Processed Crystal. Japanese Journal of Applied Physics 44, L54-L56 (2004).

2. Murakami, A. et al. Universal Processing Technique for Protein Crystals Using Pulsed UV Laser. Japanese Journal of Applied Physics 43, L873-L876 (2004). 